\title{
Assessment of the Effects of Online Linear Leak Current Compensation at Different Pacing Frequencies in a Dynamic Action Potential Clamp System
}

\author{
Alan Fabbri ${ }^{1}$, Adrianus Prins ${ }^{1}$,Teun P de Boer ${ }^{1}$ \\ ${ }^{1}$ Department of Medical Physiology, Division of Heart \& Lungs, University Medical Center Utrecht, \\ Utrecht, The Netherlands
}

\begin{abstract}
Dynamic action potential (AP) clamp (dAPC) is an electrophysiology technique that allows one to study in real time the effects of a biological current included in a computational AP model. During an experiment, the seal resistance between the cell membrane and the pipette is finite and a leak current ( $I_{\text {leak }}$ ) occurs. Its reduction is crucial to properly assess the effect of a drug. Our work aims to quantify the impact of $I_{\text {leak }}$ on a ventricular AP model and to evaluate the benefits of an online compensation.

We adopted the Ten Tusscher 2006 as human ventricular model. We used a passive model cell $\left(C_{m}=\right.$ $\left.19.8 p F, R_{\text {seal }}=500 \mathrm{MOhm}\right)$ and online compensated the leak current through a linear model. $V_{\max }, R M P$ and $A P D_{20,50,90}$ are measured at several degrees of compensation (within 0 and 100\%), at the pacing frequencies of $0.5,1$ and $2 \mathrm{~Hz}$, and compared with the scenario in which there is no connection between the model cell and the AP model.

$I_{\text {leak }}$ decreases $V_{\text {max }}$, depolarizes $R M P$ (up to $+6.1 \mathrm{mV}$ at $1 \mathrm{~Hz}$ ) and prolongs APD; a full compensation of $I_{\text {leak }}$ brings the AP biomarkers close to the open loop condition.

With this test, we show that online compensation of $I_{\text {leak }}$ is beneficial for proper assessment of AP biomarker. The correction of RMP is key, as it affects the following phases of the AP.
\end{abstract}

\section{Introduction}

Dynamic Clamp (DC) is a hybrid electrophysiology technique that allows one to link a real cell with a computational model, in real time [1]. In the dynamic Action Potential clamp (dAPC) configuration, a cell overexpressing a specific ion channel (e.g Human Embryonic Kidney (HEK, [2]) or Chinese Hamster Ovary (CHO, [3]) cells), is coupled with a whole cell action potential (AP) computational model. In this setting, the membrane potential of the computational model stimulates the ion channel overexpressed in the real cell, as would happen in a real car- diomyocyte. In turn, the ion current generated in the real cell is scaled appropriately and fed back into the computational model, thereby contributing to action potential formation, and therefore to the final AP waveform, forming a closed-loop system. As result, dAPC improves on classical electrophysiology techniques as it enables observation of the effects of a drug on the current of interest, and on the AP at the same time.

During a dAPC experiment, the resistance between the pipette and the cell membrane $\left(R_{\text {Seal }}\right)$ is finite and a leak current $\left(I_{\text {leak }}\right)$ occurs. In voltage clamp experiments, the $I_{\text {leak }}$ artifact can be corrected for during post-processing. Since dAPC is a real time technique, the $I_{\text {leak }}$ must be handled during the experiments. An online compensation avoids misleading effects on the AP waveform.

This study aims to quantify the effects of $I_{l e a k}$ and the benefits of online compensation when using a ventricular computational AP model in a dAPC experiment.

To do that, we compared five AP biomarkers (Resting Membrane Potential, RMP, Maximal upstroke Velocity, $V_{\max }$, and AP Duration at 20,50 and $90 \%$ of repolarization, $A P D_{20,50,90}$ ) for several degrees of $I_{\text {leak }}$ online compensation ( $0 \%$ (No compensation), $25 \%, 50 \%, 75 \%$ and $100 \%)$ at three pacing frequencies $(0.5 \mathrm{~Hz}, 1 \mathrm{~Hz}$ and 2 $\mathrm{Hz}$ ), adopting the open loop configuration (no $I_{\text {leak }}$ injection) as a reference.

\section{Methods}

\subsection{The dynamic AP clamp system}

In this study we used a prototype developed in our lab of the Nanion Patchliner Dynamite ${ }^{8}$ [4], adapted to the dAPC configuration and running the endocardial version of the ten Tusscher and Panfilov 2006 ventricular model (TP06, endo) [5]. The adoption of the Rush-Larsen method [6] allows one to provide a real time stable solution of TP06, splitting the set of ordinary differential equations in two groups: the time course of the gating variables is described via their analytic, exponential solutions; all the other state variables are integrated through the Forward Euler method 
with a step time of $50 \mu s$.

\subsection{The model cell and the leak current compensation}

In our dAPC experiment, the HEKA MC10 model cell (HEKA Elektronik, Germany) provides the external current that affects the final shape of the AP. The MC10 model cell consists in a passive linear parallel RC circuit $\left(R_{M C}=500 M \Omega\right.$ and $\left.C=19.8 p F\right)$. We assumed $R_{M C}=R_{\text {leak }}$, therefore, the model cell contributes to the AP shape providing $I_{\text {leak }}$. The linear components of the model cells allowed us to perform the online $I_{\text {leak }}$ compensation through a linear model [7]:

$$
\begin{gathered}
I_{\text {comp }}=\alpha_{\text {comp }} g_{\text {leak }}\left(V_{m}-E_{\text {leak }}\right) \\
I_{\text {final }}=I_{\text {leak }}-I_{\text {comp }}
\end{gathered}
$$

During the diastolic interval, the current density provided by the model cell was $\approx 8 p A / p F$, a value that wouldn't allow the TP06 to repolarize. For this reason the injected current was scaled down of a factor $1 / 10$.

\subsection{Hardware and software}

We carried out the dAPC experiments on a EPC10-USB amplifier controlled by Patchmaster v2x90.2 (HEKA Electronik, Germany). Data analysis and plots (extraction of the AP biomarkers $A P D_{20,50,90}, R M P$ and $V_{\max }$ ) are performed with custom code using Matlab 2017b (The Mathworks, Natick MA, USA).

To provide a more in depth analysis of the mechanisms responsible for the changes of the AP biomarkers, we ran simulations of the TP06 model using OpenCOR [8], reproducing the effects of the leak current with the same properties of the HEKA MC10 model cell.

\section{Results}

The effects of $I_{\text {leak }}$ on the AP morphology at $0.5,1$ and $2 \mathrm{~Hz}$ are shown in Figure 1,2 and 3. If not compensated, $I_{\text {leak }}$ depolarizes RMP (see panels C, Figure 1,2 and 3), dampens the peak (panels D) and prolongs the late repolarization phase (panels E). The online compensation of $I_{\text {leak }}$ progressively brings the AP shape back to the open loop condition, with a good superimposition when a full compensation is performed.

The biomarker extraction reveals a RMP depolarization similar for the three frequencies, with the highest variation at $1 \mathrm{~Hz}\left(R M P_{0 \%}=-78.1 \mathrm{mV}, R M P_{\text {OpenLoop }}=\right.$ $-84.2 \mathrm{mV}, \Delta R M P=+6.1 \mathrm{mV}, \Delta R M P \%=$ $+7.2 \%)$. $V_{\max }$ monotonically decreases with faster pacing $\left(\Delta V_{\max }=-18.3,-26.9\right.$ and $-30.7 \%$ at $0.5,1$ and $2 \mathrm{~Hz}$ respectively.). For a complete overview see Table 1). The APD undergoes to prolongation both in early and in late repolarization (see Table 2). Surprisingly $A P D_{20}$ lengthens between +24 and $+26.2 \%$ (at 2 and $0.5 \mathrm{~Hz}$ respectively). At first sight, indeed, one could expect a faster repolarization due to the outward $I_{\text {leak }}$, instead of a slower one. The underlying mechanisms will be explained in the Discussion section. Finally, $A P D_{50}$ and $A P D_{90}$ show smaller increases $\left(\triangle A P D_{50}=4.4 \%\right.$ at $0.5 \mathrm{~Hz}, \triangle A P D_{90}=5.5 \%$ at $2 \mathrm{~Hz}$.)

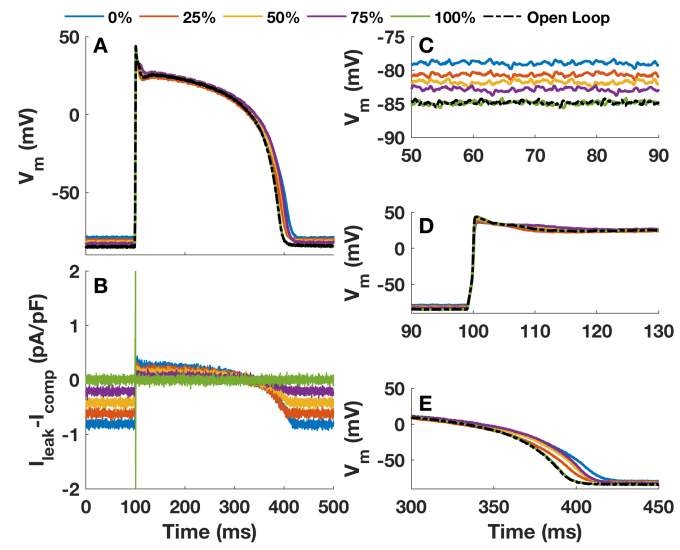

Figure 1. A-B) AP and injected current. C) RMP, D) Upstroke and peak, E) late depolarization of TP06 at 0.5 $\mathrm{Hz}$

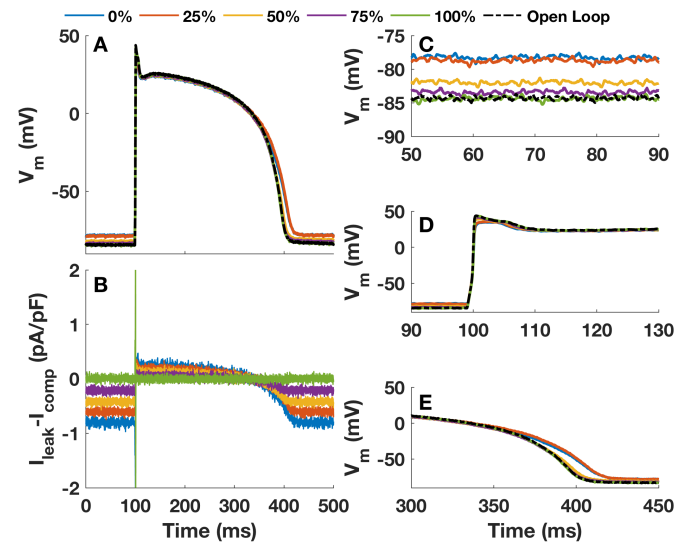

Figure 2. A-B) AP and injected current. C) RMP, D) Upstroke and peak, E) late depolarization of TP06 at $1 \mathrm{~Hz}$ 


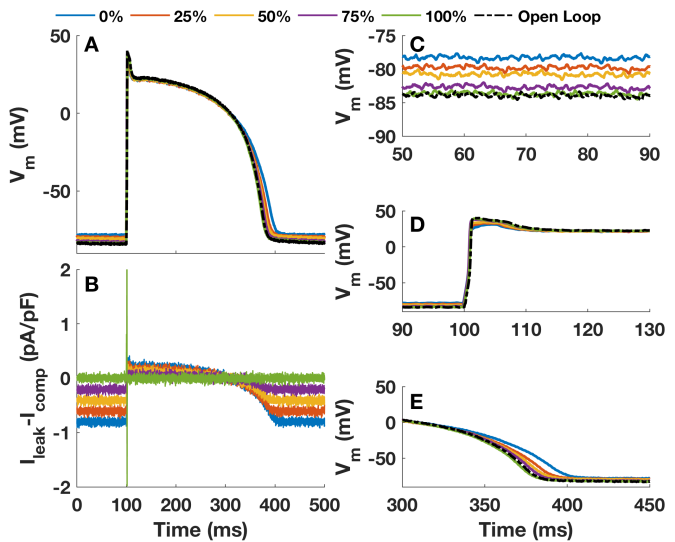

Figure 3. A-B) AP and injected current. C) RMP, D) Upstroke and peak, E) late depolarization of TP06 at $2 \mathrm{~Hz}$

Table 1. $V_{\max }$, RMP and relative changes with respect to the open loop condition at $0.5,1$ and $2 \mathrm{~Hz}$

\begin{tabular}{|c|c|c|c|c|c|}
\hline & & $\begin{array}{l}V_{\max } \\
\mathrm{V} / \mathrm{ms}\end{array}$ & $\begin{array}{c}\Delta V_{\max } \\
\%\end{array}$ & $\begin{array}{c}R M P \\
\mathrm{mV}\end{array}$ & $\begin{array}{c}\Delta R M P \\
\%\end{array}$ \\
\hline \multirow{6}{*}{$0.5 \mathrm{~Hz}$} & $0 \%$ & 248 & -18.3 & -79.0 & 6.9 \\
\hline & $25 \%$ & 268 & -11.8 & -80.6 & 4.9 \\
\hline & $50 \%$ & 291 & -4.3 & -81.7 & 3.6 \\
\hline & $75 \%$ & 308 & 1.1 & -81.8 & 3.6 \\
\hline & $100 \%$ & 304 & 0.1 & -84.9 & -0.1 \\
\hline & Open Loop & 304 & & -84.8 & \\
\hline \multirow{6}{*}{$1 \mathbf{H z}$} & $0 \%$ & 224 & -26.9 & -78.1 & 7.2 \\
\hline & $25 \%$ & 233. & -23.9 & -78.5 & 6.8 \\
\hline & $50 \%$ & 284 & -7.1 & -81.9 & 2.8 \\
\hline & $75 \%$ & 291 & -5.0 & -83.3 & 1.1 \\
\hline & $100 \%$ & 303 & -1.1 & -84.5 & -0.3 \\
\hline & Open Loop & 306 & & -84.2 & \\
\hline \multirow{6}{*}{$2 \mathrm{~Hz}$} & $0 \%$ & 187 & -30.7 & -78.2 & 6.9 \\
\hline & $25 \%$ & 214 & -20.4 & -79.7 & 5.1 \\
\hline & $50 \%$ & 231 & -14.1 & -80.8 & 3.8 \\
\hline & $75 \%$ & 260 & -3.6 & -82.7 & 1.5 \\
\hline & $100 \%$ & 265 & -1.8 & -83.8 & 0.3 \\
\hline & Open Loop & 269 & & -84.0 & \\
\hline
\end{tabular}

\section{Discussion and Conclusion}

With this study, we aimed to quantify the impact of linear $I_{\text {leak }}$ on the AP morphology of an in real time running ventricular computational model (TP06, endo [5]), integrated in a dAPC setup. To do that, we performed the online $I_{\text {leak }}$ compensation at increasing degrees (from 0 to $100 \%$ ) at $0.5,1$ and $2 \mathrm{~Hz}$, and we compared several AP biomarkers.

The results showed that $I_{\text {leak }}$ depolarizes RMP, decreases $V_{\max }$ and prolongs APD both during early and late repolarization in all the explored pacing frequencies. The full online compensation brings the biomarkers close to the open loop condition, where no current is injected.

To investigate the underlying mechanisms, we performed simulations mimicking the experimental condi-
Table 2. $A P D_{20,50,90}$ and relative changes with respect to the open loop condition at $0.5,1$ and $2 \mathrm{~Hz}$

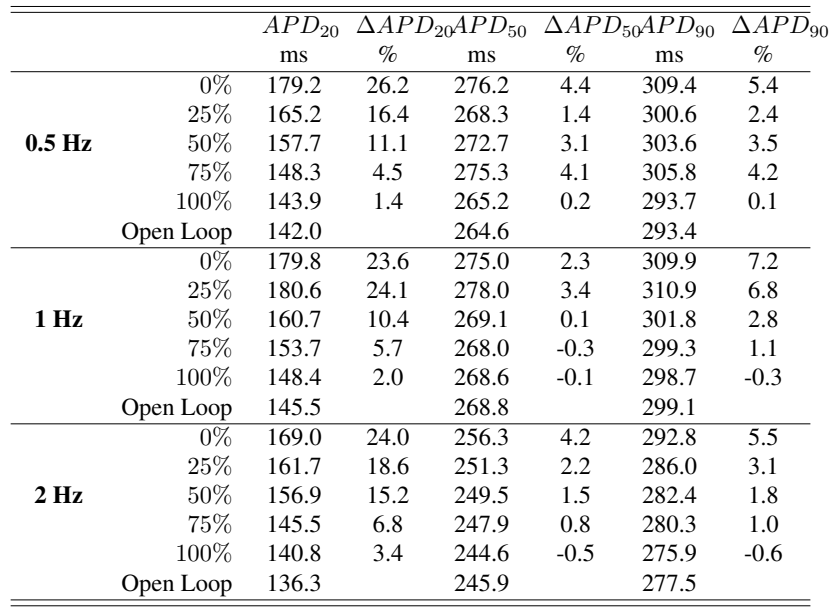

tions. Using OpenCOR [8], we observed the uncompensated $I_{\text {leak }}$ scenario (see Figure 4, red lines) with the original model (black lines), and we compared the time course of $I_{N a}$ and all the other membrane currents. The depolarized RMP inactivates $I_{N a}$ ion channels, leading to halved $I_{N a}$ peak (see Figure 4, panel B). The simulations without compensation of $I_{\text {leak }}$ showed a marked decrease of the fast (h) and slow (j) inactivation variables before and during the $I_{N a}$ peak (see Figure 4, panel E,F and G), for all the three frequencies. The activation gate $\mathrm{m}$ showed a slight decrease at 0.5 and $1 \mathrm{~Hz}$ ( -0.35 and $-2.4 \%$ respectively), whereas it undergoes to an increase of $13 \%$ at $2 \mathrm{~Hz}$, compensated by a larger decrease of $\mathrm{h}$ and $\mathrm{j}$. The reduced peak of $I_{N a}$ is therefore responsible for the decreased $V_{\max }$.

During the plateau phase, the time course of the net current in the two scenarios is similar (see Figure 4 panel C) and the repolarization rate is almost the same. This result is achieved by an interplay between the inward net current and $I_{l e a k}$ : the model reacts to $I_{\text {leak }}$ providing a slightly stronger inward current during the early stage of the repolarization (see Figure 4, panel D). $A P D_{20}$ shows an unexpected prolongation due to a smaller AP amplitude (AP peak - RMP), rather than a more intense net inward current. $I_{\text {leak }}$ slows down the late repolarization due to its inward contribution, which is responsible for a delayed and less intense repolarizing current.

The discussed results were obtained using a specific computational model (TP06). As a further step it would be fruitful to quantify the impact of $I_{\text {leak }}$ on different computational models (e.g. on the O'Hara-Rudy 2011 model [9], one of the latest and most used models). The AP waveform results from a delicate balance between a specific mix of currents, and the adoption of a different model could lead to different behaviors. 


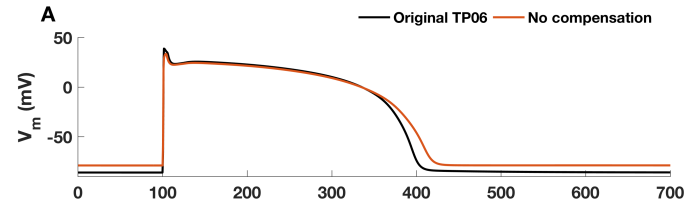

B
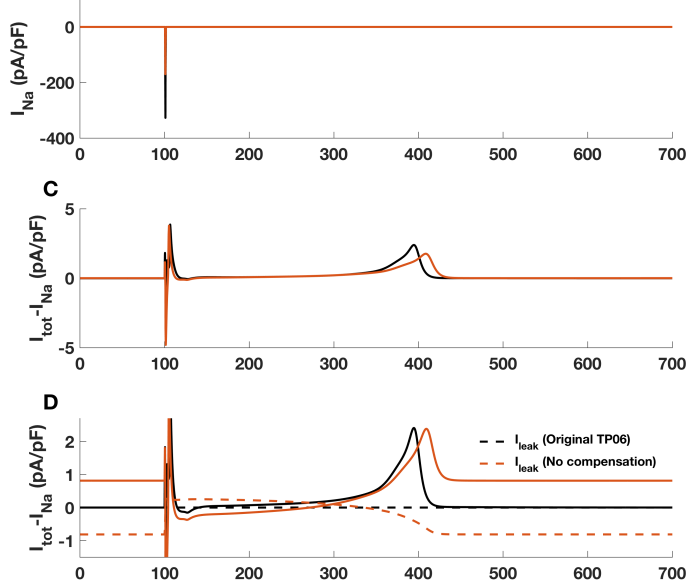

E

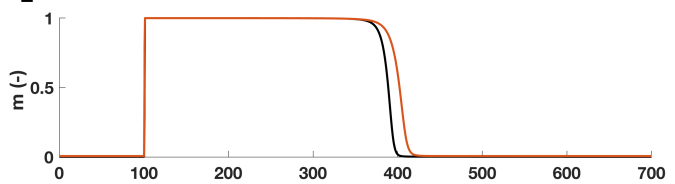

F

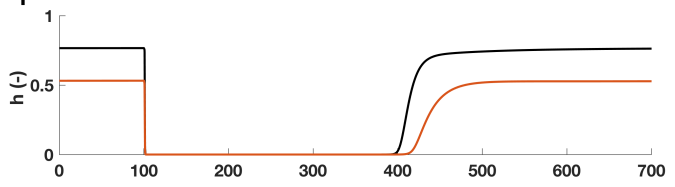

G

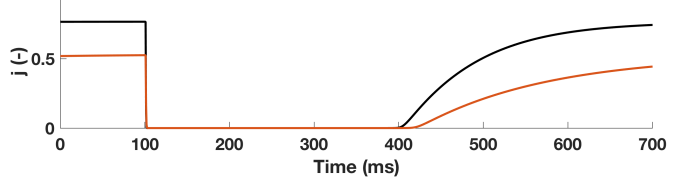

Figure 4. Underlying mechanisms responsible for changes in AP morphology. Time course of A) AP, B) $I_{N a}$, C) total net current without $\left.I_{N a}, \mathrm{D}\right)$ total net current (solid lines) and leak current (dashed lines) displayed separately. Time course of E) activation gate $\mathrm{m}, \mathrm{F}$ ) fast inactivation gate $\mathrm{h}$ and G) slow inactivation gate $\mathrm{j}$ of $I_{N a}$. Pacing frequency = $1 \mathrm{~Hz}$ but the same mechanisms hold true at 0.5 and $2 \mathrm{~Hz}$.

From the experimental perspective, our approach with the model cell allowed us to assess the effect of $I_{\text {leak }}$ with a $R_{\text {Seal }}$ value that is known and remains stable over time. This is an ideal condition that does not usually occur during an experiment with HEK or CHO cells. Our dAPC system allows the user to automatically update the current estimated value of $R_{\text {Seal }}$, which typically decreases over time. In conclusion, online compensation of $I_{\text {leak }}$ is important in dAPC experiments to avoid inaccurate results that are due to artifacts present in the experimental setup.

\section{Acknowledgments}

The authors thank Dr. Gary Mirams (The University of Nottingham) for the valuable help regarding the RushLarsen method. This research is financially supported by MKMD grants with project numbers 114021501 and 114022502 (ZonMW).

\section{References}

[1] Wilders R. Dynamic clamp: a powerful tool in cardiac electrophysiology. The Journal of Physiology 2006;576(2):349359.

[2] Thomas P, Smart TG. HEK293 cell line: A vehicle for the expression of recombinant proteins. Journal of Pharmacological and Toxicological Methods 2005;51(3):187-200.

[3] Gamper N, Stockand JD, Shapiro MS. The use of chinese hamster ovary $(\mathrm{CHO})$ cells in the study of ion channels. Journal of Pharmacological and Toxicological Methods 2005; 51(3):177-185.

[4] Becker N, Horváth A, Boer TD, Fabbri A, Grad C, Fertig N, George M, Obergrussberger A. Automated dynamic clamp for simulation of IK1 in human induced pluripotent stem cell-derived cardiomyocytes in real time using patchliner dynamite8. Current Protocols in Pharmacology 2020; 88(1):e70.

[5] ten Tusscher KHWJ, Panfilov AV. Alternans and spiral breakup in a human ventricular tissue model. American Journal of Physiology Heart and Circulatory Physiology 2006; 291(3):H1088-H1100.

[6] Marsh ME, Ziaratgahi ST, Spiteri RJ. The secrets to the success of the rush-larsen method and its generalizations. IEEE Transactions on Biomedical Engineering 2012;59(9):25062515.

[7] Ahrens-Nicklas RC, Christini DJ. Anthropomorphizing the mouse cardiac action potential via a novel dynamic clamp method. Biophysical Journal 2009;97(10):2684-2692.

[8] Garny A, Hunter PJ. OpenCOR: a modular and interoperable approach to computational biology. Frontiers in Physiology 2015;6.

[9] O’Hara T, Virág L, Varró A, Rudy Y. Simulation of the undiseased human cardiac ventricular action potential: Model formulation and experimental validation. PLOS Computational Biology 2011;7(5):e1002061.

Address for correspondence:

Alan Fabbri

Department of Medical Physiology

University Medical Center Utrecht

Yalelaan 50, 3584CM Utrecht, The Netherlands

afabbri@umcutrecht.nl 